\title{
Mechanisms of aural encoding: VI. Consonants and vowels are remembered as subsets of distinctive features*
}

\author{
RONALD ALLAN COLE \\ Lniversity of Waterloo. Waterloo, Ontario, Canada \\ RALPH NORMAN HABER \\ University of Rochester, Rochester, . New York 14627 \\ and \\ BRUCE DENNIS SALES \\ Vorthwestern Lniversity. Elanston, Mlinois 62201
}

\begin{abstract}
An attempt was made to examine the manner in which consonants and vowels are coded in short-term memory under identical recall conditions. Ss were presented with sequences of consonant-vowel digrams for serial recall. Sequences were composed of randomly presented consonants paired with /a/ or randomly presented vowels paired with id . Halle's distinctive feature system was used to generate predictions concerning the frequency of intrusion errors among phonemes. These predictions were based on the assumption that phonemes are discriminated in memory in terms of their component distinctive features. so that intrusions should most frequently occur between phonemes sharing similar distinctive features. The analysis of intrusion errors revealed that each consonant and rowel phoneme was coded in short-term memory by a particular combination of distinctive features which differed from one phoneme to another. A given phoneme was coded by the same set of distinctive features regardless of the number of syllablesin the sequence. However, distinctive feature theories were not able to predict the frequency of intrusion errors for phonemes presented in the middle serial positions of a sequence with 100? accuracy. The results of the experiment support the notion that consonant and vowel phonemes are coded in a similar manner in STM and that this coding involves the retention of a specific set of distinctive features for each phoneme.
\end{abstract}

The examination of the types of errors made in the recall of a series of sounds has revealed that phonemes are coded in short-term memory (STM) as sets of distinctive features. Wickelgren $(1965,1966)$ found that substitution errors during the ordered recall of a series of phonemes were regulated by their distinctive feature similarities. Specifically, phonemes sharing the most identical distinctive features were most of ten confused for each other in STM.

Table 1 displays the distinctive feature arrangement of seven consonant and vowel phonemes according to Halle's distinctive feature system. The vowel phonemes are uniquely described by a set of $(+)$ or $(-)$ values on four dimensions, while the consonant phonemes are uniquely described by six dimensions. For example, in this table. the vowels /i/ and /e/differ only by their feature value on the "grave" dimension. If this feature is forgotten during the serial recall of a series of phonemes, there is a 50\% probability that Ss will intrude /e/ for /i/ on the basis of the remaining feature information. Following this reasoning, the rank order of expected intrusion errors can be generated for each phoneme in a serial recall task by counting the number of features it shares with all other phonemes to be remembered.

*This research was supported in part by a research grant from the U.S. Public Health Service, No. MH-10753, to the second author. Reprint requests should be sent to Ronald Cole. Department of Psychology, University of Waterloo. Waterloo. Ontario. Canada.
Using the above procedure, Wickelgren $(1965,1966)$ measured the ability of theories of distinctive features to predict the rank order of intrusion errors among consonant and vowel phonemes in a serial recall task. For consonants, intruded phonemes were predicted with $75 \%$ accuracy by a distinctive feature system in terms of voicing, place of articulation, nasality. and openness of the vocal tract. For vowels, intrusions were predicted with $100^{\circ}$ accuracy, using a distinctive feature system in terms of the relative height and front-backness of the

Table 1

Distinctive Feature Compesition of Phonemes Used in This Experiment Based on Halle's (1962) Distinctive Feature System

\begin{tabular}{lccccccc}
\hline & \multicolumn{7}{c}{ Vowel Phonemes } \\
\cline { 2 - 7 } Dimension & $\mid \mathrm{i} /$ & /el & $\mid \mathbf{a e} /$ & $/ \mathrm{u} /$ & $\mid \mathrm{a} /$ & /0/ & $\mid \mathrm{J} /$ \\
\hline Flat & - & - & - & + & - & + & + \\
Compact & - & - & + & - & + & - & + \\
Grave & + & - & - & + & - & - & - \\
Diffuse & - & - & - & + & + & + & + \\
\hline
\end{tabular}

Consonant Phonemes

\begin{tabular}{lccccccc}
\cline { 2 - 6 } Dimension & $/ \mathrm{d} /$ & $\mid \mathrm{s} /$ & $/ \mathrm{m} /$ & $\mid \theta_{i}$ & $\mid \mathrm{n} /$ & $/ \mathbf{s} /$ & $\mid \mathrm{p} /$ \\
\hline Grave & - & - & + & - & - & - & + \\
Diffuse & + & - & + & + & + & + & + \\
Strident & - & + & - & - & - & + & - \\
Nasal & - & - & + & - & + & - & - \\
Continuant & - & + & - & + & - & + & - \\
Voiced & + & - & + & - & + & - & - \\
\hline
\end{tabular}


tongue. On the basis of these results. Wickelgren concluded that phonemes are coded in short-term memory as sets of distinctive features which may be forgotten independently of each other. When such forgetting occurs, phonemes having similar distinctive features are intruded for the forgotten phoneme.

Sales. Haber, and Cole $(1968,1969)$ have found that only certain combinations of distinctive features perfectly predicted intrusion errors for vowel phonemes forgotten in a serial recall task. For example, only the dimensions "grave" plus "diffuse" in Table 1 might perfectly predict the frequency with which other vowels were intruded for $/ \mathrm{i} /$, while the dimensions "flat" plus "grave" would perfectly predict the frequency with which other vowel phonemes were intruded for $/ \mathrm{e} /$. On the basis of these data. Sales. Haber, and Cole concluded that. of the total population of phonemic distinctive features available to $S$. only certain subsets of these features were used to code a vowel phoneme in short-term memory.

The present experiment was designed to examine distinctive feature coding for consonants and vowels under identical recall conditions. It was predicted that the frequency of intrusion errors for both consonants and vowels would be perfectly predicted by some combination of the total population of distinctive features used to describe each phoneme.

The experiment was also designed to investigate the manner in which distinctive feature coding is affected by the serial position in which a phoneme is heard. It has long been known that it is easier to remember the first and last items as compared to the middle items in a series. If the probability of forgetting a phoneme in the middle of a sequence is caused by the loss of feature information in these positions, then it may be predicted that distinctive feature theories will be less accurate in predicting intrusion errors for phonemes in the middle serial positions of a sequence. This prediction is based on the reasoning that intrusions should be less orderly when many rather than few distinctive features are forgotten for a given phoneme. When only one coded feature is forgotten for a phoneme, there are few phonemes that will be intruded on the basis of the remaining features and intrusions will be orderly and predictable. However, when several (or all) features are forgotten for that phoneme, the $\mathrm{S}$ retains very little information about the forgotten phoneme, so that intrusion errors will tend to be randomly determined. Therefore. if feature forgetting is more likely to occur in the middle positions of a sequence, then distinctive feature theories should less accurately predict intrusion errors for these positions.

\section{METHOD}

\section{Subjects}

One hundred and twenty students from the University of California at Riverside served as $\mathrm{Ss}$. These $\mathrm{Ss}$ received one credit tow ard their grade in an introductory psychology course. Each $S$ was pretested to ascertain whether he could discriminate and respond to each of the stimuli. Sil potential Ss were eliminated for their inability to perform the $/ a / / 2$, distinction.

\section{Stimuli}

The seven consonant and vowel phonemes presented in Table 1 were used as stimuli. Consonant phonemes were followed by $/ a /$. while vowel phonemes were preceded by $/ d /$ so that all phonemes were heard as members of a consonant-vowel syllable. These phonemes were chosen because their distinctive feature arrangements allowed a range of comparisons from phonemes sharing many similar features (e.g.e $/ \mathrm{m} / \mathrm{vs} / \mathrm{n} / \mathrm{f} / \mathrm{i} / \mathrm{vs}$ /e/) to phonemes sharing few features (e.g.. $/ \mathrm{d} / \mathrm{vs} / \mathrm{s} / . / \mathrm{i} / \mathrm{vs} / \mathrm{\partial} /$ ).

Since the procedure for determining which distinctive features best predict the frequency of intrusion errors for any given phoneme involves a series of post hoc analyses. it was necessary to determine the reliability of this procedure. Therefore, each consonant and vowel phoneme in Table 1 was presented for recall in three different experimental groups which differed only by the number of phonemes presented in each sequence. 1 Thus. Ss were assigned to one of six experimental groups. These groups consisted of sequences of 5,6 . or 7 consonants paired with/a/ (C5, C6, C7) or sequences of 5,6 , or 7 vowels paired with $/ \mathrm{d} /$ (V5, V6. V7).

\section{Procedure}

In order to insure that intrusion errors resulted from forgetting and not from misperceptions, $10 \mathrm{Ss}$ were individually: tested for their ability to discriminate among the stimuli in a sequence. These Ss. who did not participate in the memory: experiment, were told to press a response key as soon as they heard a specified consonant or vowel phoneme. Each $S$ was tested on all 98 trials and was required to identify each consonant and vowel phoneme. For these $10 \mathrm{Ss}$. oniy eight misperceptions occurred, and $S$ s reported that these were due to attentional factors.

Ninety-eight recall sequences were constructed according to the following rules: (a) each phoneme occurred only once per sequence. (b) each phoneme occurred an equal number of times in each serial position across sequences, (c) each phoneme was presented an equal number of times with all other phonemes. The 98 sequences in each group were randomly ordered and recorded by the first author at the rate of 3 phonemes every $2 \mathrm{sec}$. Thus, the $20 \mathrm{Ss}$ in each experimental group heard the sequences in the same order. Trials were presented via Wollensak tape recorder. After each trial, E stopped the tape recorder and transcribed S's report. Responses were also recorded on another tape recorder.

Each $S$ was instructed to report back the sounds which he heard in the order in which he heard them. $S$ was allowed to either guess or say "blank" if he had forgotten a sound.

\section{RESULTS}

\section{Phonemes as Combinations of F eatures}

Analyses reported in this section were performed in order to determine whether specific combinations of distinctive features could be used to predict the frequency of intrusion errors among forgotten phonemes.

In order to examine the factors regulating intrusion errors, matrices were first constructed that displayed the total number of times all $S$ s in a group intruded any given phoneme with any other phoneme. For example. 
in Table 2, when /d/ was presented in sequences of five consonants, it was intruded as $/ \mathrm{m} / 68$ times and intruded as is/ 29 times. If the scores in Table 2 are rank ordered within each row, the result is Table 3 , which displays the rank order of intruded phonemes for each presented phoneme. However. Table 3 is subject to response bias in two ways. First, since omission errors occur with unequal frequency for the different phonemes, the phonemes are effectively presented an unequal number of times. Second, responses that occur very often will appear high in the rank ordering of all phonemes. Thus, if $S$ has a bias to say /i/ whenever he forgets a vowel phoneme, then /i/ will be intruded most often regardless of the presented phoneme.

To control for the first source of response bias, conditional probabilities of occurrence were calculated for each phoneme. This was accomplished by summing the entries in each row in Table 2 and then dividing each entry in the row by its sum. The result of this manipulation is Table 4. which gives the conditional probability of intruding any two phonemes. Table 4 is free from response bias due to unequal omission errors among the phonemes. Finally, response bias due to frequently recalled phonemes may be eliminated by rank ordering the columms in Table 4 . The resulting tables present the rank order of presented phonemes for each intruded phoneme based on their conditional probabilities of occurrence. "This comparison within a column ... equates the response bias-that is the S's bias to emit any particular sound, independent of its strength in short-term memory [Wickelgren, 1966, p. 396]."

Following the above procedure, conditional probabilities were computed for the phonemes in each experimental group. Although these tables (not shown) actually display the rank order of presented sounds for each intruded phoneme, the data are referred to as intrusions for the sake of conceptual simplicity.

In order to test the hypothesis that phonemes are coded in STM as sets of binary distinctive features, Halle's distinctive feature system was used to predict the rank ordering of intrusion errors for each phoneme in this experiment. These predictions were based on the assumption that phonemes sharing the most identical distinctive features are most likely to be intruded for each other in STM. For example. on the dimensions "flat" and "grave," it can be seen from Table 1 that /i/ shares two features with $/ \mathrm{e} /$, one with $/ \mathbf{a} /, / \mathrm{u} /, / \mathrm{a} / . / \mathrm{o} /$, and no features with $10 /$. If Ss code $/ i$ in STM by the features "flat" and "grave," then the rank order of intrusion errors should be $/ \mathrm{e} />/ \mathbf{x} / . / \mathrm{u} /, \mathrm{a} / . / \mathrm{o} /,>/ \mathrm{o} /$. This ordering yields nine distinct predictions: $|\mathrm{e} />| \mathfrak{a} \mid$, $\mid \mathrm{u} /, / \mathrm{a} /, / \mathrm{o} / \mathrm{/} / \mathrm{o} /$, and $/ \mathrm{a} / . / \mathrm{u} / . / \mathrm{a} /, / \mathrm{o} />/ \mathrm{o} /$. If the actual rank order of intrusions in this experiment is identical to the predicted order of intruded phonemes. then the ratio (number of intrusions correctly. predicted)/(total possible number of possible predictions) would be 9/9. or 100\%. Rank-order predictions for single dimensions and all combinations of dimensions in
Table 2

Intrusion Matrix for Phonemes in the C5 Condition*

\begin{tabular}{|c|c|c|c|c|c|c|c|}
\hline \multirow{2}{*}{$\begin{array}{l}\text { Presented } \\
\text { Phonemes }\end{array}$} & \multicolumn{7}{|c|}{ Intruded Phonemes } \\
\hline & $|d|$ & $|\boldsymbol{s}|$ & $\mathrm{m}$ & $1 \theta i$ & $i n$ & 5 & p \\
\hline $\begin{array}{l}|\mathrm{d}| \\
|\mathrm{s}| \\
|\mathrm{m}| \\
|\theta| \\
|\mathrm{n}| \\
|\mathrm{s}| \\
|\mathrm{p}|\end{array}$ & $\begin{array}{r}101 \\
96 \\
116 \\
105 \\
79 \\
96\end{array}$ & $\begin{array}{r}57 \\
66 \\
98 \\
81 \\
229 \\
74\end{array}$ & $\begin{array}{r}68 \\
58 \\
\\
81 \\
144 \\
78 \\
80\end{array}$ & $\begin{array}{r}108 \\
95 \\
60 \\
\\
65 \\
113 \\
65\end{array}$ & $\begin{array}{r}100 \\
53 \\
127 \\
103\end{array}$ & $\begin{array}{l}29 \\
73 \\
26 \\
46 \\
32 \\
\\
33\end{array}$ & $\begin{array}{r}87 \\
61 \\
82 \\
104 \\
87 \\
97\end{array}$ \\
\hline
\end{tabular}

*Data summed over 20 Ss in all five serial positions

Table 3

Rank Ordering of Intruded Phonemes for Each Presented Phoneme Based on the Scores in Table 2 (Group C5)

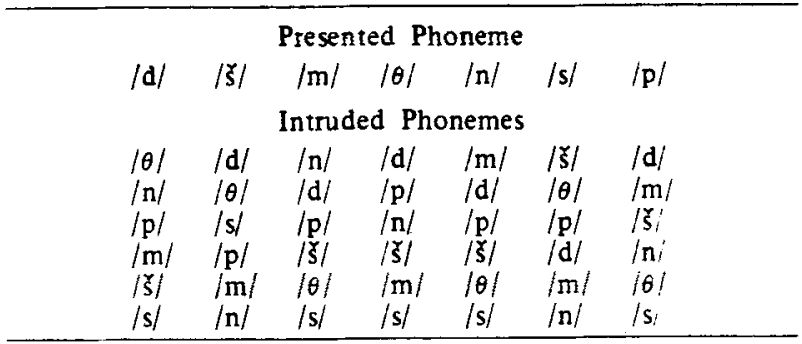

Table 4

Conditional Probability of Intruding Any Two Phonemes in the C5 Condition*

\begin{tabular}{|c|c|c|c|c|c|c|c|}
\hline \multirow{2}{*}{$\begin{array}{l}\text { Presented } \\
\text { Phonemes }\end{array}$} & \multicolumn{7}{|c|}{ Intruded Phonemes } \\
\hline & $/ \mathrm{d} /$ & $|\xi|$ & $/ \mathrm{m} /$ & $|\theta|$ & $n i^{\prime}$ & $\mathbf{s i}_{i}^{\prime}$ & $\mathrm{p} !$ \\
\hline$/ \mathrm{d} /$ & & .127 & .151 & .240 & .222 & .064 & .194 \\
\hline $\mid \check{s} !$ & .229 & & .131 & .215 & .120 & .165 & .138 \\
\hline$/ \mathrm{m} /$ & .210 & .144 & & .131 & .278 & .057 & .179 \\
\hline$|\theta|$ & .212 & .179 & .148 & & .188 & .084 & .188 \\
\hline$|\mathbf{n}|$ & .204 & .157 & .280 & .126 & & .062 & .169 \\
\hline$|s|$ & .117 & .341 & .114 & .168 & .114 & & .144 \\
\hline$/ \mathrm{p} /$ & .230 & .178 & .192 & .156 & .163 & .079 & \\
\hline
\end{tabular}

*Data summed over 20 Ss in all five serial positions

Table 1 were applied to the intrusion data for consonants and vowels in each experimental group.

The test of individual dimensions is particularly interesting because it provides a measure of the relative strength of each dimension in STM. Predictions based on the "shared-feature" hypothesis for individual dimensions require the additional assumption that $a$ feature may be maintained in memory while the phoneme with which it was associated is no longer remembered.

Considering all features taken together (the measure used by Wickelgren). Halle's system predicted consonant intrusions with $81.6^{\circ} \mathrm{c}$ accuracy and vowel intrusions with $84.6 \%$ accuracy. Thus, both consonants and vowel intrusions are predicted with approximately equal accuracy by a distinctive feature theory when phonemes are recalled under identical conditions.

Rank-order predictions were also tested for all combinations of distinctive feature dimensions for each 
Table 5

Those Dimensions. or Their Combinations. Which Best Predict the Frequency of Intrusion Errors for Consonant Phonemes in Sequences of Five. Six, or Seven Syllables*

\begin{tabular}{|c|c|c|c|c|}
\hline \multirow{2}{*}{ Phonemes } & \multirow{2}{*}{$\begin{array}{c}\text { Best } \\
\text { Predicting } \\
\text { Dimen- } \\
\text { sions }\end{array}$} & \multicolumn{3}{|c|}{ Sequence Length } \\
\hline & & $\mathrm{Cs}$ & $\mathrm{C} 6$ & $\mathrm{C7}$ \\
\hline$/ \mathrm{d} /$ & $\begin{array}{l}\text { S } \\
\text { NC }\end{array}$ & $\begin{array}{l}6 / 8 \\
5 / 5\end{array}$ & $\begin{array}{l}8 / 8 \\
4 / 5\end{array}$ & $\begin{array}{l}8 / 8 \\
5 / 5\end{array}$ \\
\hline$|\zeta|$ & $\begin{array}{l}\text { S } \\
C \\
\text { DS } \\
\text { DC } \\
\text { SC }\end{array}$ & $\begin{array}{l}5 / 5 \\
8 / 8 \\
5 / 5 \\
8 / 8 \\
9 / 9\end{array}$ & $\begin{array}{l}5 / 5 \\
8 / 8 \\
5 / 5 \\
8 / 8 \\
9 / 9\end{array}$ & $\begin{array}{l}5 / 5 \\
8 / 8 \\
5 / 5 \\
8 / 8 \\
9 / 9\end{array}$ \\
\hline$/ \mathrm{m} /$ & $\begin{array}{l}\mathbf{N} \\
\mathrm{C} \\
\mathrm{GN} \\
\mathrm{GV} \\
\mathrm{NC}\end{array}$ & $\begin{array}{c}5 / 5 \\
9 / 9 \\
8 / 8 \\
9 / 9 \\
11 / 11\end{array}$ & $\begin{array}{c}5 / 5 \\
9 / 9 \\
8 / 8 \\
9 / 9 \\
11 / 11\end{array}$ & $\begin{array}{c}5 / 5 \\
9 / 9 \\
8 / 8 \\
9 / 9 \\
11 / 11\end{array}$ \\
\hline$|\theta|$ & $\begin{array}{l}\boldsymbol{N} \\
\text { GSN } \\
\text { SNC }\end{array}$ & $\begin{array}{l}8 / 8 \\
8 / 9 \\
8 / 8\end{array}$ & $\begin{array}{l}8 / 8 \\
9 / 9 \\
8 / 8\end{array}$ & $\begin{array}{l}7 / 8 \\
9 / 9 \\
7 / 8\end{array}$ \\
\hline$/ \mathbf{n} /$ & $\begin{array}{l}\mathrm{N} \\
\mathrm{V} \\
\mathrm{NV} \\
\text { GNCV }\end{array}$ & $\begin{array}{l}5 / 5 \\
8 / 8 \\
9 / 9 \\
8 / 8\end{array}$ & $\begin{array}{l}5 / 5 \\
8 / 8 \\
9 / 9 \\
8 / 8\end{array}$ & $\begin{array}{l}5 / 5 \\
8 / 8 \\
9 / 9 \\
8 / 8\end{array}$ \\
\hline$|s|$ & $\begin{array}{l}\text { S } \\
\text { DS } \\
\text { SV } \\
\text { DSV }\end{array}$ & $\begin{array}{c}5 / 5 \\
5 / 5 \\
11 / 11 \\
9 / 9\end{array}$ & $\begin{array}{c}5 / 5 \\
5 / 5 \\
11 / 11 \\
9 / 9\end{array}$ & $\begin{array}{c}5 / 5 \\
5 / 5 \\
10 / 11 \\
8 / 9\end{array}$ \\
\hline$|\mathrm{p}|$ & $\begin{array}{l}\text { S } \\
\text { SN } \\
\text { SCV } \\
\text { SNCV }\end{array}$ & $\begin{array}{l}8 / 8 \\
8 / 8 \\
8 / 8 \\
8 / 8\end{array}$ & $\begin{array}{l}7 / 8 \\
8 / 8 \\
7 / 8 \\
8 / 8\end{array}$ & $\begin{array}{l}8 / 8 \\
7 / 8 \\
8 / 8 \\
7 / 8\end{array}$ \\
\hline
\end{tabular}

*Fractions describe the ratio of correct predictions to possible correct predictions. $G=$ grave, $D=$ diffuse. $S=$ strident. $. N=$ nasal. $C=$ continuant. $V^{r}=$ voiced.

phoneme in Table 1. This analysis revealed that some combination of distinctive features perfectly predicted the frequency of intrusion errors for each phoneme in each experimental group. Further, the combination of distinctive features which perfectly predicted the frequency of intrusion errors was often found to differ from one phoneme to another.

To determine whether the same combination of dimensions yielded the best prediction in each group, all of the subsets of features were rank ordered by their success in predicting the frequency of intrusion errors for each phoneme. Spearman rank-order correlations were then computed between the different groups for each phoneme. The resulting correlations were significant for each phoneme $(\mathrm{p}<.05)$. For consonants, these correlations ranged from .40 for $/ \mathrm{s} /$ to .67 for $/ \mathrm{m} /$. For vowels, values ranged from .44 for $/ \mathrm{s} /$ to .88 for $/ \mathrm{i} /$, $/ \mathrm{e} /$, and $/ \mathrm{u} /$. Thus, the same distinctive features best predicted the frequency of intrusion errors for each phoneme in sequences of 5,6 , or 7 syllables. Tables 5 and 6 display those combinations of distinctive features which consistently predicted the frequency of intrusion errors for phonemes in each experimental group.

The finding that the same combinations of features best predicted the rank order of intruded errors in each experimental group strongly suggests that $S$ s code phonemes as sets of distinctive features. Given that $S$ codes a phoneme in STM as a particular set of distinctive features, the question that may be asked is: What determines which combination will be used? One possibility is that $S$ chooses the subset of features that most uniquely specifies each phoneme in relation to the other phonemes to be remembered. This possibility predicts that for each phoneme. $S$ will use those dimensions sharing the fewest identical features with the other phonemes to be remembered. Following this reasoning, the "uniqueness" of each combination of features was calculated for each phoneme according to the formula: uniqueness $=$ (number of dimensions considered)/(number of shared features with all other phonemes on these dimensions). The ratio for each combination of features was computed and then converted to a percentage. Combinations of features having the highest percentages were considered most unique.

All combinations of features were rank ordered for each phoneme by their score on the uniqueness measure. This rank ordering by "uniqueness" was compared to the rank ordering of dimensions by their ability to predict the pattern of intrusion errors. If $S$ uses the most unique combination of fatures to code a phoneme. ihen that same combination of features should most accurately predict the pattern of intrusion errors for that

Table 6

Those Dimensions, or Their Combinations, Which Best Predict the Frequency of Intrusion Errors for Vowel Phonemes in Sequences of Five, Six, or Seven Syllables*

\begin{tabular}{|c|c|c|c|c|}
\hline \multirow{2}{*}{ Phonemes } & \multirow{2}{*}{$\begin{array}{c}\text { Best } \\
\text { Predicting } \\
\text { Dimen- } \\
\text { sions }\end{array}$} & \multicolumn{3}{|c|}{ Sequence Length } \\
\hline & & V5 & V6 & V7 \\
\hline /i/ & $\begin{array}{l}\text { C } \\
\text { D } \\
\text { CD } \\
\text { FCD }\end{array}$ & $\begin{array}{c}9 / 9 \\
5 / 5 \\
11 / 11 \\
11 / 11\end{array}$ & $\begin{array}{c}9 / 9 \\
5 / 5 \\
11 / 11 \\
11 / 11\end{array}$ & $\begin{array}{c}9 / 9 \\
5 / 5 \\
11 / 11 \\
11 / 11\end{array}$ \\
\hline$|e|$ & $\begin{array}{l}\text { G } \\
\text { FC } \\
C G\end{array}$ & $\begin{array}{c}8 / 8 \\
9 / 9 \\
11 / 11\end{array}$ & $\begin{array}{c}7 / 8 \\
9 / 9 \\
11 / 11\end{array}$ & $\begin{array}{c}8 / 8 \\
8 / 9 \\
10 / 11\end{array}$ \\
\hline$|\mathbf{x}|$ & D & $7 / 8$ & $8 / 8$ & $8 / 8$ \\
\hline$/ \mathrm{u} /$ & $\mathrm{C}$ & $9 / 9$ & $9 / 9$ & $9 / 9$ \\
\hline$|a|$ & $\begin{array}{l}\mathrm{C} \\
\text { FCG }\end{array}$ & $\begin{array}{l}8 / 8 \\
8 / 8\end{array}$ & $\begin{array}{l}8 / 8 \\
8 / 8\end{array}$ & $\begin{array}{l}8 / 8 \\
8 / 8\end{array}$ \\
\hline $10 /$ & FCD & $9 / 9$ & $9 / 9$ & $9 / 9$ \\
\hline $\mid 2 /$ & $\begin{array}{l}\mathrm{D} \\
\text { FCD } \\
\text { CDG }\end{array}$ & $\begin{array}{c}8 / 8 \\
11 / 11 \\
13 / 13\end{array}$ & $\begin{array}{c}8 / 8 \\
11 / 11 \\
13 / 13\end{array}$ & $\begin{array}{c}8 / 8 \\
11 / 11 \\
13 / 13\end{array}$ \\
\hline
\end{tabular}

*Fractions describe the ratio of correct predictions to possible correct predictions. $F=$ flat. $C=$ compact. $D=$ diffuse. $G=$ grave. 
phoneme. Therefore, orderings by uniqueness and by ability to predict intrusions should correlate.

Spearman rank-order correlations revealed that the uniqueness of a combination of features is significantly correlated with the ability of these features to predict intrusion errors for four out of seven consonant phonemes $(p<.05)$, while this correlation is significant in only two out of seven cases for the vowel phonemes $(p<.05)$. Although the model tested is successful in predicting feature-coding strategies for consonants, it does not have sufficient generality to account for distinctive feature-coding strategies for votvels.

\section{Distinctive Features and Serial Position}

A final set of analyses was performed to assess the ability of Halle's distinctive feature theory to predict the pattern of intrusion errors for phonemes in the different serial positions. For these analyses, the rank order of presented sounds in each experimental group was calculated for each intruded sound for each serial position, according to the procedures outlined above. This manipulation yielded 5.6, or 7 separate confusion matrices for each group, depending upon the number of phonemes presented for recall in that group.

The ability of Halle's distinctive feature system to predict the rank order of intruded phonemes for groups of each sequence length is shown in Fig. 1. Accuracy of prediction in this figure is summed over all phonemes at each serial position. Figure 1 reveals that Halle's theory generally predicts the frequency of intrusion errors slightly better in the initial and final positions than in the middle serial positions of a sequence. However, even in the middle serial positions, prediction is consistently greater than $50 \%$, which is chance prediction.

\section{DISCUSSION}

No differences were observed in the manner in which consonants and vowels were coded in STM. The frequency of intrusion errors for any given consonant or vowel phoneme in this experiment was accurately predicted by the same distinctive features in groups of different sequence length. Further, both consonant and vowel phonemes revealed an increase in feature forgetting in the middle serial positions of the sequence which led to slightly less accurate prediction of the intrusion errors in these positions.

It is interesting to note that similar coding strategies are used to remember consonants and vowels in STM, since several lines of evidence suggest that consonants and vowels are perceived quite differently. Experiments in dichotic listening have revealed that, when Ss are simultaneously presented with a pair of consonants. one to each ear. there is a clear superiority in identifying those presented to the right ear (Shankweiler \& Studdert-Kennedy. 1967). (This effect is reversed for Ss with a dominant right hemisphere.) However. pairs of

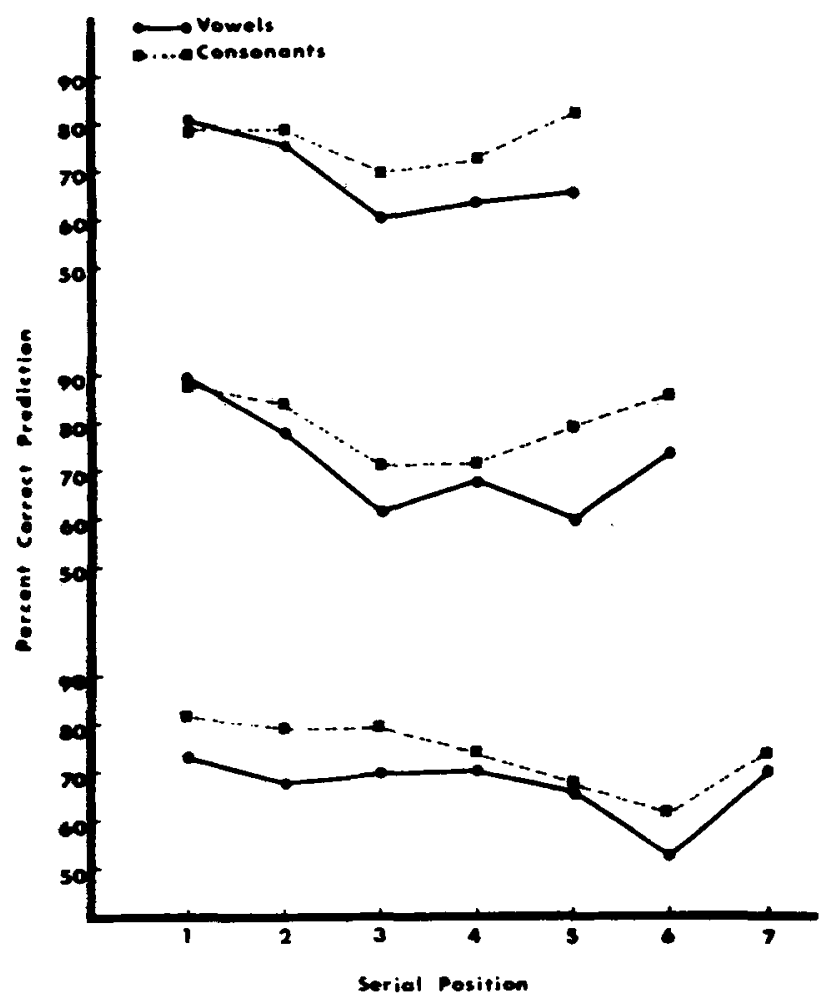

Fig. 1. Accuracy with which Halle's distinctive feature system predicts the frequency of intrusion errors for phonemes in each serial position for groups of 5,6. or 7 syllables. Prediction scores are summed over 7 phonemes and $20 \mathrm{Ss}$ in each group.

dichotically presented vowels either reveal no laterality effect or show a slight advantage for those presented to the left ear.

Experiments at Haskins Laboratories using synthetically produced speech have demonstrated that perception of certain consonants is categorical. while vowel perception is continuous (Liberman. Cooper. Shankweiler. \& Studdert-Kennedy. 1967). For stop consonants, listeners perceive a range of acoustic stimuli as a single phoneme and are unable to discriminate acoustic variations within this range. On the other hand, vowel perception is continuous in that the continuous change of an acoustic cue results in a corresponding perceptual change in the vowel sound.

On the basis of these data, Liberman et al have concluded that consonants are perceived in terms of a special code. while vowels are processed as complex nonspeech sounds (such as a musical tone). Whether or not consonants and vowels are analyzed differently during recognition. it is clear that they are both coded as sets of distinctive features in STM.

Distinctive feature theories require approximately five binary distinctive features to describe all English vowels and six binary features to describe all English consonants. This experiment revealed that Ss use only certain combinations of these features to code a phoneme in shorteterm memors. and the particular 
combination of features used may differ from one phoneme to another.

The coding strategy used by $S$ to maintain an ordered series of phonemes in memory appears to involve the retention of one or more "preferred" distinctive features for each phoneme. For example, when $S$ is presented with $/ \mathrm{m} /$. it is likely that he retains the features "nasal" and "continuant," since this combination of features predicts the intrusion errors for $/ \mathrm{m} /$ with $100 \%$ accuracy in all three experimental conditions. On the other hand, when $\mathrm{n} /$ is heard, he is likely to retain the features "nasal" and "voiced," since this combination of dimensions predicts the intrusion data for $/ \mathrm{n} /$ with $100 \%$ accuracy in the three conditions in which $/ \mathrm{n} /$ occurred.

By using this coding strategy, $S$ is able to discriminate among ' $m$ and $/ n^{\prime}$ using only two features to remember each phoneme. It is unlikely that $S$ would use only the "nasality" features to code both ' $m$, and $/ \mathrm{n} /$ in STM, since this code would not permit $S$ to differentiate among these two phonemes. However, if S uses "nasal" plus "continuant" to code ' $\mathrm{m}$ ' and "nasal" plus "voiced" to code $/ \mathrm{n} /$, he is able to discriminate $/ \mathrm{m} /$ and $/ \mathrm{n} /$ from each other and from all other phonemes. using a minimal number of distinctive features to remember each phoneme. Seen in this light, the strategy of coding a phoneme in STM as a limited set of distinctive features may be an efficient way of remembering a sequence of phonemes.

It is still not known what determines the set of features that were used to code a particular phoneme in STM. There was a tendency for $S$ to code a consonant phoneme by those distinctive features which most uniquely identify a phoneme in relation to a given population of consonant phonemes. However, the limited success of this model suggests that other, as yet unknown, factors are determining the choice of features used to code a phoneme in STM.

\section{REFERENCES}

Halle, M. Phonology in a generative grammar. Word, 1962. 18. 54.72.

Liberman. A. II.. Cooper. F. S.. Shankweiler. D. P.. \& Studdert-Kennedy. M. Perception of the speech code. Psy'chological Review, 1967. 74, 431-461.

Sales. B. D.. Haber. R. N.. \& Cole, R. A. Mechanisms of aural encoding: III. Distinctive features fón vonicls. Perception \& Psychophysics, 1968. 4, 321-327.

Sales, B. D., Haber, R. N.. \& Cole. R. A. Mechanisms of aural encoding: IV. Hear-say, see-write interactions. Perception \& Psychophysics, 1969, 6B. 385-390.

Shankweiler, D., \& Studdert-Kennedy. M. Identification of consonants and vowels presented to left and right ears. Quarterly Journal of Experimental Psychology. 1967. 19. 59.63.

Wickelgren. W. A. Distinctive features and errors in short-term menory for English vowels. Journal of the Acoustical Society of America. 1965. 38, 583-588.

Wickelgren. W. A. Distinctive features and errors in short-term memory for English consonants. Journal of the Acoustical Society of America, 1966, 39, 388-398.

\section{NOTE}

1. Wickelgren (1966) and Sales, Haber, and Cole (1968) have found that sequence length does not affect the pattern of intrusion errors among consonants or vowels in STM.

(Received for publication March 6. 1972.) 